\title{
Asymptotic Expansions of Integrals of Certain Rapidly Oscillating Functions
}

\author{
By U. Banerjee, L. J. Lardy, and A. Lutoborski
}

\begin{abstract}
An expansion in terms of powers of $m^{-1}$ is given for integrals of the form $\int_{0}^{1} f(x) \bar{u}(m x) d x$, where $m$ is a positive integer, $\bar{w}(m x)$ is an integrable rapidly oscillating function with period $m^{-1}$, and $f(x)$ is a smooth function.
\end{abstract}

1. Introduction. Asymptotic expansions in terms of powers of $\mathrm{m}^{-1}$ of the Fourier coefficients

$$
\int_{0}^{1} f(x) \cos (2 \pi m x) d x \text { and } \int_{0}^{1} f(x) \sin (2 \pi m x) d x
$$

of a smooth function $f(x)$ are well known. For example, they are discussed in [5] and [6]. The purpose of this note is to derive similar asymptotic expansions for integrals with more general periodic rapidly oscillating factors in the integrand. Such integrals occur as energy integrals in the study of elastic properties of composite materials [2].

Let $w$ be a real function belonging to $L^{1}[0,1]$, and let it be extended by periodicity to all of $\mathbf{R}$ with the extended function denoted by $\bar{w}$. It will be assumed that the moments of $w$,

$$
\mathscr{M}_{k}=\mathscr{M}_{k}(w)=\int_{0}^{1} y^{k} w(y) d y, \quad k \geqslant 0,
$$

are known. We shall consider integrals of the form

$$
I^{m}(f)=\int_{0}^{1} f(x) \bar{w}(m x) d x
$$

where $m$ is a positive integer and $f(x)$ is smooth.

The following result on the limiting value of $I^{m}(f)$ is classical [2, p. 9].

Proposition 1.1. If $w \in L^{1}[0,1]$ and $f \in L^{\infty}[0,1]$, then

$$
\lim _{m \rightarrow \infty} I^{m}(f)=\mathscr{M}_{0}(w) \int_{0}^{1} f(x) d x .
$$

It is natural to search for an asymptotic expansion of $I^{m}(f)$ in terms of powers of $m^{-1}$. Such an expansion is developed in Section 3. Section 2 is devoted to preliminary results.

Received March 12, 1986; revised August 6, 1986.

1980 Mathematics Subject Classification (1985 Revision). Primary 41A55; Secondary 65D30.

1987 American Mathematical Society $0025-5718 / 87 \$ 1.00+\$ .25$ per page 
2. Expansions of Euler-Maclaurin Type. When the integral of a product is expanded using integration by parts, certain auxiliary functions and their mean values arise naturally as in $[4$, p. 210]. In this section these are introduced and are represented in terms of Bernoulli functions and Bernoulli numbers. The Bernoulli numbers are denoted by $B_{n}$, the Bernoulli polynomials by $B_{n}(x)$, and the Bernoulli functions, the periodic extensions from $[0,1)$ to all of $\mathbf{R}$ of the polynomials $B_{n}(x)$, are denoted by $\bar{B}_{n}(x),[1$, p. 804].

Definition 2.1. Set $p_{0}(y)=w(y)$ and $\alpha_{0}=\mathscr{M}_{0}(w)$. For $y \in[0,1]$, define recursively for $j \geqslant 0$,

$$
\begin{aligned}
p_{j+1}(y) & =\int_{0}^{y}\left[\alpha_{j}-p_{j}(u)\right] d u, \\
\alpha_{j+1} & =\int_{0}^{1} p_{j+1}(y) d y .
\end{aligned}
$$

Clearly,

$$
p_{j}(0)=0=p_{j}(1), \quad j \geqslant 1,
$$

and

$$
p_{j}^{(i)}(y)=(-1)^{i-1}\left[\alpha_{j-i}-p_{j-i}(y)\right], \quad 1 \leqslant i \leqslant j .
$$

Proposition 2.2. If $g \in C^{k+1}[0,1]$, then

$$
\int_{0}^{1} g(y) w(y) d y=\sum_{j=0}^{k} \alpha_{j} \int_{0}^{1} g^{(j)}(y) d y+\int_{0}^{1} p_{k+1}(y) g^{(k+1)}(y) d y .
$$

Proof. For $0 \leqslant j \leqslant k$, in view of (2.1), (2.2), and (2.3), integration by parts yields

$$
\int_{0}^{1}\left[\alpha_{j}-p_{j}(y)\right] g^{(j)}(y) d y=-\int_{0}^{1} p_{j+1}(y) g^{(j+1)}(y) d y .
$$

Thus,

$$
\sum_{j=0}^{k} \int_{0}^{!}\left[\alpha_{j}-p_{j}(y)\right] g^{(j)}(y) d y=-\sum_{j=0}^{k} \int_{0}^{1} p_{j+1}(y) g^{(j+1)}(y) d y .
$$

After cancelling terms common to both sides, this last equation simplifies to (2.5).

This expression for the integral of a product of two functions can also be developed using similar ideas from [4, p. 210].

Formula (2.5) for $w(y)=\cos y$ (or $\sin y$ ) is well known and was used in [5] and [6].

Proposition 2.3. The coefficients $\alpha_{k}$ are related to the moments $\mathscr{M}_{k}$ by the formula

$$
\alpha_{k}=\sum_{j=0}^{k} \frac{B_{k-j}}{j !(k-j) !} \mathscr{M}_{j}, \quad k \geqslant 0 .
$$

Proof. Consider formula (2.5) with $g(y)=B_{k}(y)$. From [3, p. 48], this gives

$$
\int_{0}^{1} B_{k}(y) w(y) d y=\alpha_{k} k !
$$

Formula (2.6) now follows by substituting for $B_{k}(y)$ the expression $[4$, p. 6]

$$
B_{k}(y)=\sum_{j=0}^{k} \frac{k !}{j !(k-j) !} B_{k-j} y^{j} .
$$

Numerical values of the coefficients in (2.6) for $0 \leqslant k \leqslant 5$ are shown in Table 1.1. 
TABLE 1.1

\begin{tabular}{c}
$\alpha_{k}$ in terms of the $\mathscr{M}_{j}$ \\
\hline$\alpha_{0}=\mathscr{M}_{0}$ \\
$\alpha_{1}=-\frac{1}{2} \mathscr{M}_{0}+\mathscr{M}_{1}$ \\
$\alpha_{2}=\frac{1}{12} \mathscr{M}_{0}-\frac{1}{2} \mathscr{M}_{1}+\frac{1}{2} \mathscr{M}_{2}$ \\
$\alpha_{3}=\frac{1}{12} \mathscr{M}_{1}-\frac{1}{4} \mathscr{M}_{2}+\frac{1}{6} \mathscr{M}_{3}$ \\
$\alpha_{4}=-\frac{1}{720} \mathscr{M}_{0}+\frac{1}{24} \mathscr{M}_{2}-\frac{1}{12} \mathscr{M}_{3}+\frac{1}{24} \mathscr{M}_{4}$ \\
$\alpha_{5}=-\frac{1}{720} \mathscr{M}_{1}+\frac{1}{72} \mathscr{M}_{3}-\frac{1}{48} \mathscr{M}_{4}+\frac{1}{120} \mathscr{M}_{5}$ \\
\hline
\end{tabular}

It is also easy to obtain a recursive relation between the coefficients $\alpha_{j}$ and the moments $\mathscr{M}_{j}$ which might be useful in some situations. These formulas are given in the next proposition.

Proposition 2.4. The coefficients $\alpha_{k}$ are related to the moments $\mathscr{M}_{j}$ by the following formulas:

For $k$ odd,

$$
\alpha_{k}=-2^{-k}\left[\frac{1}{k !} \sum_{j=0}^{k}(-2)^{j}\left(\begin{array}{l}
k \\
j
\end{array}\right) \mathscr{M}_{j}+\sum_{j=0}^{(k-3) / 2} \frac{2^{2 j+1}}{(k-2 j) !} \alpha_{2 j+1}\right]
$$

For $k$ even,

$$
\alpha_{k}=2^{-k}\left[\frac{1}{k !} \sum_{j=0}^{k}(-2)^{j}\left(\begin{array}{l}
k \\
j
\end{array}\right) \mathscr{M}_{j}-\sum_{j=0}^{(k-2) / 2} \frac{2^{2 j}}{(k-2 j+1) !} \alpha_{2 j}\right] .
$$

Proof. Let $g(y)=\left(y-\frac{1}{2}\right)^{k}$. On the one hand, by expanding the binomial power, the integral on the left in (2.5) can be expressed as

$$
\int_{0}^{1} w(y)\left(y-\frac{1}{2}\right)^{k} d y=\sum_{j=0}^{k}\left(\begin{array}{l}
k \\
j
\end{array}\right)\left(-\frac{1}{2}\right)^{k-j} \mathscr{M}_{j} .
$$

On the other hand, for this choice of $g(y)$, the expansion on the right in (2.5) reduces to

$$
\sum_{j=0}^{k} \alpha_{j} \int_{0}^{1} g^{(j)}(y) d y=\sum_{j=0}^{k} \alpha_{j} \frac{k !}{(k-j+1) !} 2^{j-k-1}\left(1+(-1)^{k-j}\right) .
$$

Equating the expressions on the right in (2.9) and (2.10), solving the resulting equation for $\alpha_{k}$, and considering the separate cases of $k$ odd and $k$ even, will yield formulas (2.7) and (2.8) respectively.

Proposition 2.5. The functions $p_{k}(y)$ have the following integral representations

$$
p_{k}(y)=\alpha_{k}+\frac{(-1)^{k}}{k !} \int_{0}^{1} \bar{B}_{k}(y-u)\left[\alpha_{0}-w(u)\right] d u .
$$

Proof. The arguments in [4, pp. 15-17] remain valid under the more general hypothesis that the function $f$ has a derivative of order $\nu$ in $L^{1}[0,1]$, because the operations of integration by parts are justified also in this case. Thus, from [4, p. 17] 
with $\nu=k$ and $f$ replaced by $p_{k}$, and using (2.3) and (2.4),

$$
\frac{1}{k !} \int_{0}^{1} p_{k}^{(k)}(u) \bar{B}_{k}(y-u) d u=-p_{k}(y)+\int_{0}^{1} p_{k}(u) d u .
$$

In view of (2.4) and (2.2), formula (2.11) follows immediately.

Proposition 2.6. The functions $p_{k}(y)$ are given in terms of the coefficients $\alpha_{j}$ by the formula

$$
p_{k}(y)=\sum_{j=1}^{k} \alpha_{k-j} \frac{(-1)^{j-1}}{j !} y^{j}+\frac{(-1)^{k}}{(k-1) !} \int_{0}^{y}(y-u)^{k-1} w(u) d u .
$$

Proof. The proof is by induction on $k$.

Note first that

$$
\int_{0}^{y} \int_{0}^{u}(u-v)^{k-1} w(v) d v d u=\frac{1}{k} \int_{0}^{y}(y-u)^{k} w(u) d u .
$$

Formula (2.12) is easily verified for $k=1$.

If (2.12) holds for some $k \geqslant 1$, then

$$
\begin{aligned}
p_{k+1}(y) & =\int_{0}^{y}\left[\alpha_{k}-p_{k}(u)\right] d u \\
& =\alpha_{k} y-\sum_{j=1}^{k} \alpha_{k-j} \frac{(-1)^{j-1}}{(j+1) !} y^{j+1}-\frac{(-1)^{k}}{(k-1) !} \int_{0}^{y} \int_{0}^{u}(u-v)^{k-1} w(v) d v d u \\
& =\sum_{j=1}^{k+1} \alpha_{k+1-j} \frac{(-1)^{j-1}}{j !} y^{j}+\frac{(-1)^{k+1}}{k !} \int_{0}^{y}(y-u)^{k} w(u) d u .
\end{aligned}
$$

Thus (2.12) is valid for $k+1$.

3. The Asymptotic Expansion Formula. In this section an asymptotic expansion formula is established for integrals of the form (1.1). Let $m$ be a positive integer and set $x_{i}=i / m$ for $0 \leqslant i \leqslant m$.

THEOREM 3.1. If $w \in L^{1}[0,1]$ and $f \in C^{k+1}[0,1]$, then

$$
\int_{0}^{1} f(x) \bar{w}(m x) d x=\sum_{j=0}^{k} m^{-j} \alpha_{j} \int_{0}^{1} f^{(j)}(x) d x+R_{k+1, m},
$$

where for $k \geqslant 1$,

$$
\left|R_{k+1, m}\right| \leqslant 8(2 \pi m)^{-(k+1)}\|w\|_{1} \zeta(k+1)\left\|f^{(k+1)}\right\|_{\infty},
$$

with $\zeta(k+1)$ the Riemann zeta function, and for $k=0$,

$$
\left|R_{1, m}\right| \leqslant 2 m^{-1}\|w\|_{1}\left\|f^{(1)}\right\|_{\infty} .
$$

Proof. First write

$$
\int_{0}^{1} f(x) \bar{w}(m x) d x=\sum_{i=0}^{m-1} \int_{x_{i}}^{x_{i+1}} f(x) \bar{w}(m x) d x .
$$

Making the change of variable $y=m\left(x-x_{i}\right)$, setting $g_{i}(y)=f\left(x_{i}+m^{-1} y\right)$, and using the definition of $\bar{w}(m x)$, we obtain

$$
\int_{0}^{1} f(x) \bar{w}(m x) d x=\sum_{i=0}^{m-1} m^{-1} \int_{0}^{1} g_{i}(y) w(y) d y .
$$


Since $g_{i}(y) \in C^{k+1}[0,1]$ and $g_{i}^{(j)}(y)=m^{-j} f^{(j)}\left(x_{i}+m^{-1} y\right)$, from (2.5),

$$
\int_{0}^{1} g_{i}(y) w(y) d y=\sum_{j=0}^{k} \alpha_{j} \int_{0}^{1} g_{i}^{(j)}(y) d y+\int_{0}^{1} p_{k+1}(y) g_{i}^{(k+1)}(y) d y .
$$

Changing the variable of integration back to $x$ yields

$$
\begin{aligned}
\int_{0}^{1} g_{i}(y) w(y) d y= & \sum_{j=0}^{k} \alpha_{j} m^{1-j} \int_{x_{i}}^{x_{i+1}} f^{(j)}(x) d x \\
& +m^{-k} \int_{x_{i}}^{x_{i+1}} p_{k+1}\left(m\left(x-x_{i}\right)\right) f^{(k+1)}(x) d x .
\end{aligned}
$$

Let the function $p_{k+1}$ be extended from $[0,1]$ to all of $\mathbf{R}$ by periodicity, with the extended function denoted by $\bar{p}_{k+1}$. Substituting (3.5) into (3.4) then yields

$$
\begin{aligned}
\int_{0}^{1} f(x) \bar{w}(m x) d x= & \sum_{j=0}^{k} \alpha_{j} m^{-j} \int_{0}^{1} f^{(j)}(x) d x \\
& +m^{-(k+1)} \int_{0}^{1} \bar{p}_{k+1}(m x) f^{(k+1)}(x) d x .
\end{aligned}
$$

The bound (3.3) is immediate.

To establish the bound (3.2), note that

$$
\left|R_{k+1, m}\right| \leqslant m^{-(k+1)}\left\|p_{k+1}\right\|_{\infty} \int_{0}^{1}\left|f^{(k+1)}(y)\right| d y .
$$

By (2.11),

$$
\begin{aligned}
\left|p_{k+1}(y)\right| & =\left|\int_{0}^{y}\left[\alpha_{k}-p_{k}(v)\right] d v\right|=\frac{1}{k !}\left|\int_{0}^{y} \int_{0}^{1} \bar{B}_{k}(v-u)\left[\alpha_{0}-w(u)\right] d u d v\right| \\
& \leqslant \frac{1}{k !} \int_{0}^{1}\left|\alpha_{0}-w(u)\right|\left|\int_{0}^{y} \bar{B}_{k}(v-u) d v\right| d u .
\end{aligned}
$$

Using the Fourier series representation for $\bar{B}_{k}$ [1, p. 805], it follows that

$$
\left|\int_{0}^{y} \bar{B}_{k}(v-u) d v\right| \leqslant 4 k !(2 \pi)^{-(k+1)} \sum_{j=1}^{\infty} \frac{1}{j^{k+1}} .
$$

Thus,

$$
\left|p_{k+1}(y)\right| \leqslant 8(2 \pi)^{-(k+1)} \zeta(k+1)\|w\|_{1}
$$

which completes the proof.

COROllaRy 3.2. If $f \in C^{\infty}[0,1]$ and if there exists a constant $C$ such that $\left\|f^{(k)}\right\|_{\infty} \leqslant C$ for all $k>0$, then for $m>1$,

$$
\int_{0}^{1} f(x) \bar{w}(m x) d x=\sum_{j=0}^{\infty} m^{-j} \alpha_{j} \int_{0}^{1} f^{(j)}(x) d x .
$$

For functions $f(x)$ whose derivatives at 0 and 1 are readily computed, it would be tempting to use the finite series

$$
\alpha_{0} \int_{0}^{1} f(x) d x+\sum_{j=1}^{k} m^{-j} \alpha_{j}\left[f^{(j-1)}(1)-f^{(j-1)}(0)\right]
$$


as an approximation of $I^{m}(f)$. However, in the absence of an acceptable bound on the remainder $R_{k+1, m}$, this can be dangerous. Indeed, in general situations, it is not uncommon to have $R_{k+1, m}$ diverge as $k \rightarrow \infty$. An excellent discussion of the pitfalls in using asymptotic expansions directly in numerical integration is given by Lyness in [5].

The form of the asymptotic expansion for the case of an oscillating factor which comes from a function originally defined on $[0, Y]$ and a smooth factor defined on $[c, d]$ is given in the following corollary.

Corollary 3.3. Let $\tilde{w} \in L^{1}[0, Y], f \in C^{k+1}[c, d]$, and let $\alpha_{j}$ and $p_{j}$ be generated via (2.1) and (2.2) with $w(y / Y)=\tilde{w}(y)$. Then

$$
\begin{aligned}
\int_{c}^{d} f(x) \overline{\tilde{w}}(m x) d x= & \sum_{j=0}^{k}\left(\frac{Y}{m}\right)^{j} \alpha_{j} \int_{c}^{d} f^{(j)}(x) d x \\
& +\left(\frac{Y}{m}\right)^{k+1} \int_{c}^{d} \tilde{p}_{k+1}(x) f^{(k+1)}(x) d x,
\end{aligned}
$$

where $\overline{\tilde{w}}$ is the periodic extension of $\tilde{w}$ from $[0, Y]$ to $\mathbf{R}$ and

$$
\tilde{p}_{k+1}(x)=p_{k+1}\left(\frac{m}{Y}(x-c)-i\right)
$$

for $c+i Y / m \leqslant x \leqslant c+(i+1) Y / m$.

Finally, we note that the expansion formula (3.1) is related very closely to the corresponding formula that results when $w$ is replaced by its image under the orthogonal projection of $L^{2}[0,1]$ onto the subspace of polynomials on $[0,1]$ of degree at most $k$. To see this, let $\Pi_{k}$ denote the orthogonal projection. Then $\Pi_{k} w$ can be written as

$$
\left(\Pi_{k} w\right)(y)=\sum_{j=0}^{k} \gamma_{k, j}\left(y-\frac{1}{2}\right)^{j}
$$

Since

$$
\left\langle\left(\Pi_{k} w\right)(y),\left(y-\frac{1}{2}\right)^{i}\right\rangle=\left\langle w(y),\left(y-\frac{1}{2}\right)^{i}\right\rangle \text { for } 0 \leqslant i \leqslant k,
$$

the coefficients $\gamma_{k, j}$ are related to the moments $\mathscr{M}_{j}(w)$ by the system (see (2.9))

$$
\sum_{j=0}^{k} \gamma_{k, j} \int_{0}^{1}\left(y-\frac{1}{2}\right)^{i+j} d y=\left(-\frac{1}{2}\right)^{i} \sum_{j=0}^{i}(-2)^{j}\left(\begin{array}{l}
i \\
j
\end{array}\right) \mathscr{M}_{j}(w), \quad 0 \leqslant i \leqslant k .
$$

Also, for $0 \leqslant j \leqslant k$,

$$
\mathscr{M}_{j}\left(\Pi_{k} w\right)=\int_{0}^{1} y^{j}\left(\Pi_{k^{w}}\right)(y) d y=\int_{0}^{1} y^{j} w(y) d y=\mathscr{M}_{j}(w) .
$$

In view of the linear relation between $\alpha_{j}$ and $\mathscr{M}_{j}$, if follows that the quantities $\alpha_{j}$ derived from $\Pi_{k} w$ agree with those derived from $w$, i.e., $\alpha_{j}\left(\Pi_{k} w\right)=\alpha_{j}(w)$. Thus from (2.5),

$$
\int_{0}^{1}\left(\Pi_{k} w\right)(y) g(y) d y=\sum_{j=0}^{k} \alpha_{j} \int_{0}^{1} g^{(j)}(y) d y+\int_{0}^{1} \tilde{p}_{k+1}(y) g^{(k+1)}(y) d y,
$$


where $\tilde{p}_{k+1}$ is derived from $\Pi_{k} w$ just as $p_{k+1}$ is derived from $w$. This shows that the functions $\bar{w}$ and $\overline{\Pi_{k} w}$ produce identical expansions (3.1) except for the remainder terms, which are different, though of the same order in $\mathrm{m}^{-1}$.

Department of Mathematics

Syracuse University

Syracuse, New York 13244-1150

1. M. Abramowitz \& I. A. Stegun, Handbook of Mathematical Functions, Dover, New York, 1972.

2. A. Bensoussan, J. L. Lions \& G. Papanicolaou, Asymptotic Analysis for Periodic Structures, North-Holland, Amsterdam, 1978.

3. P. J. DAVIS, Interpolation and Approximation, Dover, New York, 1975.

4. V. I. KryLOV, Approximate Calculation of Integrals, Macmillan, New York, 1962.

5. J. N. LyNESS, "The calculation of Fourier coefficients by the Möbius inversion of the Poisson summation formula, Part I. Functions whose early derivatives are continuous," Math. Comp., v. 24, 1970, pp. $101-135$.

6. H. J. StetTer, "Numerical approximation of Fourier transforms," Numer. Math., v. 8, 1966, pp. $235-249$. 